\title{
Public Transportation Management System based on GPS/WiFi and Open Street Maps
}

\author{
Saed Tarapiah \\ Telecommunication Engineering Dept. \\ An-Najah National University \\ Nablus,Palestine
}

\author{
Shadi Atalla \\ Lavoro Autonomo (LA) \\ Torino \\ Italy
}

\begin{abstract}
Information technology (IT) has transformed many industries, from education to health care to government, and is now in the early stages of transforming transportation systems. Transportation faces many issues like high accidents rate in general, and much more rate in developing countries due to the lack of proper infrastructure for roads, is one of the reasons for these crashes. In this project we focus on public transportation vehicles - such as buses, and mini-buses -, where the goal of the project is to design and deploy a smart/intelligent unit attached to public vehicles by using embedded microcontroller and sensors and empowering them to communicate with each other through wireless technologies. The proposed Offline Intelligent Public Transportation Management System will play a major role in reducing risks and high accidents rate, whereas it can increase the traveler satisfactions and convenience. Here, we propose a method, software as well as a framework as enabling technologies to for evaluation, planning and future improvement the public transportation system. Our system even though can be as whole or parts can be applied all over the world we mostly target developing countries. This limitation mostly appear by consider off-shelf technologies such as WiFi, GPS and Open Street Maps (OSM).
\end{abstract}

\section{Keywords-ITS; GPS; WiFi; Transportation; OSM}

\section{INTRODUCTION}

Intelligent Transportation Systems (ITS) have received much attention in recent years in academia, industry and standardization entities due to their wide impact on people's life as their scope to provide vital applications and services to improve transportation safety and mobility and to optimize the usage of available transportation resources and time. ITS applications and services rely on advanced technologies to be deployed and distributed among the intelligent infrastructure systems and vehicles system. Mainly, these technologies include but not limited to physical world perceive technologies that able to perform real world measurements and convert them into the digital world, processing and storage capabilities that operate on the digital measurements by storing, analyzing them and communication technologies include wired and wireless technologies to exchange the collected data among the vehicles them self and also to their infrastructure and vice versa.

GPS tracking devices stand at the core of the enabling perceive technologies for ITS applications and services. Indeed, the number of vehicles' GPS-enabled On-board Unit has sharply increased due to their vital and beneficial rules for both the vehicles and the drivers. GPS-based services include invehicle satellite navigation, vehicle security system, accident notification and tracking along with monitoring to name few.
Although most of GPS-based application depend on real time information collection, the historic GPS collected data intrinsically has great potential for further offline based application such as compute the journey speed, congestion monitoring, accidents deep analysis such as accidents reason and driver's behavior. Preliminary results of our study are reported in [1] This project aims to build an open framework that focuses on traffic and vehicular data for enhancing Public Transportation Management System (PTMS) efficiency in terms of analysis and planning. The proposed framework consists of four main phases namely user data collection, transmission, data analysis and decision making.

The first phase, data collection, uses off-the-shelf hardware components in order to build Smart on-Board Unit (SBU) that is fitted in to the public Transport Vehicles such as buses and mini-buses. SBU endowed with limited processing capabilities, temporary and persistent memory such as EPROM, GPS sensor and $\mathrm{WiFi}$ module to transfer the collected information to database storage. GPS tracking devices collect information regarding the vehicle such as the vehicles geographical location (i.e. longitude and latitude), speed and the driving direction at regular intervals of time [1]. The design of this phase relies on integrating multi-sensor capabilities together, in order to increase of the range of possible application that may serve the public transportation system such as collecting the pollution level along the road segments.

In the second phase, transmission, since we are storing the data from the earlier phase onto SBU, here we are interested to transfer the information to a back-end server. We are using a WiFi-based throwbox to the access point located in the main bus station which act as a gateway connected through the internet to the back-end server. The these data is in central database server based on a trip ID that is unique which is consist of triplet the vehicle ID, trip start time and end time.

The third phase, data analyses, the core of this phase is to inject the bus geographical location at a given timestamp along the trip on a digitized map such as open street maps (OSM) each street segment has different attributes such as the street category (pedestrian, highway or motorway) each category has a maximum allowed speed attribute. Here, we record the number of times a vehicle violates these speed limits and the corresponding violation time durations. The resulted information is compared to predefined threshold and limits which allows the system to decide whether the vehicle violated the traffic regulations or not at a given trip. After running our model for long enough periods, we expect that tracking 
and ticketing system can be fed by authorized department which can be utilized to get clear view about infrastructure which can be used for developing and planning to improve the infrastructure on some field or apply some regulations which will aim to reduce traffic accidents.

OSM, which provides free geographical information, is sometimes referred to as a map version of Wikipedia. Its data additions, updates, and corrections are made available by its participants.

\section{RELATED WORK}

This section explores a selected tracking fleet of vehicles solutions appeared on academic and research works so far. This work considers only solutions span a whole system for tacking a group of vehicles. In general tracking systems composed of two parts, the first part is on-Board Unit attached to moving vehicles, whilst the second part is a central application to collect, to process and to visualize useful reports. This section attempts to classify the considered related work based in different criteria. Each criteria requires intrinsic requirements to build the system.

Table I presents and compares selected related works based on the following criteria.

The first criteria (namely Type) differentiates between online and offline tracking systems. While online systems require the on-Board Unit to have permanent (available everytime and everywhere) connection with the central application. In the contrary Offline systems aggregate the collected data on a local storage unit and communicates with the central application only when the communication link available such as WiFi. The Offline systems focus on historical data processing and visualizing the generated reports( such as track a vehicle over a digital map). The offline system data transmission can be handled manually such as removing the on-Board Unit form the vehicle and connected it to a PC and transfer the data. Finally, some systems can be considered as online, offline or combined (online and offline) solutions.

The second criteria (namely Smart Unit Type) here we differentiate between different types of hardware used for build on-Board Unit for the tracking system. Three main units hardware are considered, first Commercial unit available in the market, second option could be available smart Phone with GPS and wireless links, the last option is the customized unit( where authors provide design and implementations in the considered work).

The third criteria (namely connection Type) which states the communication channel used by the on-Board Unit to transfer the collected data. Such connections could be any cellular connection or combination of them such as GPRS, SMS and 3G for simplicity we call it cellular connection.

Last criteria (namely Features) this part we make comparison between different tracking systems such as : features:

1) Visual Vehicles' Tracking : the ability to project the vehicle trip onto geographic digital map.

2) Instantaneous Vehicles Speed : Reporting the vehicle speed along the travelling track.

\begin{tabular}{|c|c|c|}
\hline Data Element & Type & Size[Bytes] \\
\hline \hline Vehicle ID & String & 8 \\
\hline longitude & float & 4 \\
\hline latitude & float & 4 \\
\hline speed & float & 4 \\
\hline direction & byte & 1 \\
\hline Timestamp & integer & 4 \\
\hline
\end{tabular}

TABLE II: Single GPS Data Record

3) Alerting: Send alerts to the driver such as breaking maximum speed on specific road segment while driving.

4) Geo-fencing: Identify geometric shapes over the digital map where the driver have to avoid while moving.

5) Geo-Casting: Sending Alerting information to specific central office and the vehicles in proximity of the vehicle generating accident or alrm situations.

\section{System Model PHASES AND ENABling TECHNOLOGIES}

\section{A. GPS Data Collection}

GPS tracker data used in this work is supplied from SBU fitted to public transportation vehicles. This data consist of one record for each instance a vehicle reported its position. Each record includes Vehicle ID, vehicle type, position coordinates(longitude and latitude), speed, date and time, direction.

Table II illustrates the GPS data record of interest. The Data type is described by $\mathrm{C}$ programming language notations. SBU stores the while GPS data record but the Vehicle ID. The total size of each locally stored GPS recoded equals 17 Bytes. With second-to-second data logging the SBU requires $17 \times 60 \times 60=61200$ Bytes of local storage to accommodate one hour of GPS recording.

The Vehicle ID is an unique identifier but is anonymous and does not include information about the driver identity.

If the vehicle moves away from the source bus station (the wireless connection with access point will not be available) then SBU will perceive and store the GPS recodes data into a local storage for off-line data logging. If the vehicle arrives close to the destination bus station, (the wireless connection reestablished again) the SBU will send the collected trip data to the gateway storage in the bus-station and will delete it is local copy for the sake on disk space.

SBU is equipped with motion detection sensory device. Which means that SBU can detect anonymously and automatically if it is moving or in stationary state. Through this way the SBU will log GPS data if it is moving and it will go to sleep mode and stop collecting GPS data if it detect that it is in stationary state for the last 5 minutes and when the SBU moves again to will resume collecting the GPS data.

\section{B. GPS Data Transmission}

This includes the tools and mechanisms to transfer the perviously collected GPS data to the back-end server for permanent storage in database system. Furthermore, it also considers the intermediates transfer from one place to another till arrive it final back-end server. 


\begin{tabular}{|c|c|c|c|c|c|c|c|c|}
\hline \multirow{2}{*}{ Reference(s) } & \multirow{2}{*}{ Type } & \multirow{2}{*}{ Smart Unit Type } & \multirow{2}{*}{ Connection Type } & \multicolumn{4}{|c|}{ Features } \\
\cline { 5 - 8 } & & & & Tracking & Speed & Alerting & Geo-fencing & Geo-Casting \\
\hline$[2],[3]$ & Online & Custom Unit & GPRS & Yes & Yes & Yes & No & No \\
\hline$[4]$ & Online & Custom Unit & GPRS and SMS & Yes & No & Yes & No & Yes \\
\hline$[5]$ & Online & Custom Unit & GPRS & Yes & Yes & No & No & No \\
\hline$[6]$ & Online & Custom Unit & SMS & Yes & Yes & No & No & No \\
\hline$[7]$ & Combined & Custom Unit & GPRS & Yes & Yes & Yes & No & No \\
\hline$[8]$ & Combined & Custom Unit & SMS & Yes & No & No & No & No \\
\hline$[9]$ & Combined & Commercial Unit & GPRS and SMS & Yes & Yes & Yes & Yes & No \\
\hline$[10]$ & Combined & Smart Phone & GPRS, 3G and SMS & Yes & Yes & Yes & No & No \\
\hline$[11]$ & Online & Smart Phone & Cellular & Yes & Yes & No & No & No \\
\hline
\end{tabular}

TABLE I: Features of Others Vehicle Monitoring \& Tracking Systems

The transmission technologies vary among many communication option depending the intended application for instance real time tracking application will require an instance and permanent mobile connection to the back-end server or any intermediate stage which always have direct connection to the back-end server. Cellular connections (2G, 3G, and LTE) are the conventional shapes of communication to this purposes. Whilst for non real time application, in particular this work, this ease a lot the communication challenges. Thus, the communication options include the aforementioned ones plus other options like vehicle mobility which is well known as Delay Tolerant Networks (DTN). DTN exploits the vehicle mobility to transfer the GPS collected data from one point to another. [12].

\section{GPS Data Analysis}

1) Injecting GPS Data into Map: We utilized Open Street Map [13] to inject the travel path along the trip by using the javascript library Open Layer [14].

2) Measurements and Statistics : Different statistics can be carried out on the collected data with different criteria it cloud be statistics on a given trip, a give route, a give time period, or on a given vehicle behaviour. Driver's Average Speed distribution - String -For each trip we calculate the average speed along the trip after having number of runs/trips we cloud identify the best fit distribution that describes the average along given path per driver/vehicle moreover since we consider a give road followed by every public transportation vehicle(mandatory) so the total trip distance in kilome ter is fixed (i.e. $25 \mathrm{~km}$ for we consider path Toulkarem/Nablus). But the trip traveling duration directly proportional to the average speed such information cloud be used to estimate the vehicle arrival at the given bus stop and cloud be used for enhancing the travel scheduling to be more realistic. pathway's Average Speed distribution - String - For each trip we calculate the average speed along the trip after having number of runs/trips we cloud identify the best fit distribution that describes the average along given path per route/pathway.

\section{ARCHITECTURE AND IMPLEMENTATIONS}

This section depicts the high level architecture( as shown in Figure 1) of the proposed system by identifying the main building blocks which consists the SBU which is mounted and attached to the vehicles. In addition, the Web based application running on the back-end server is introduced.

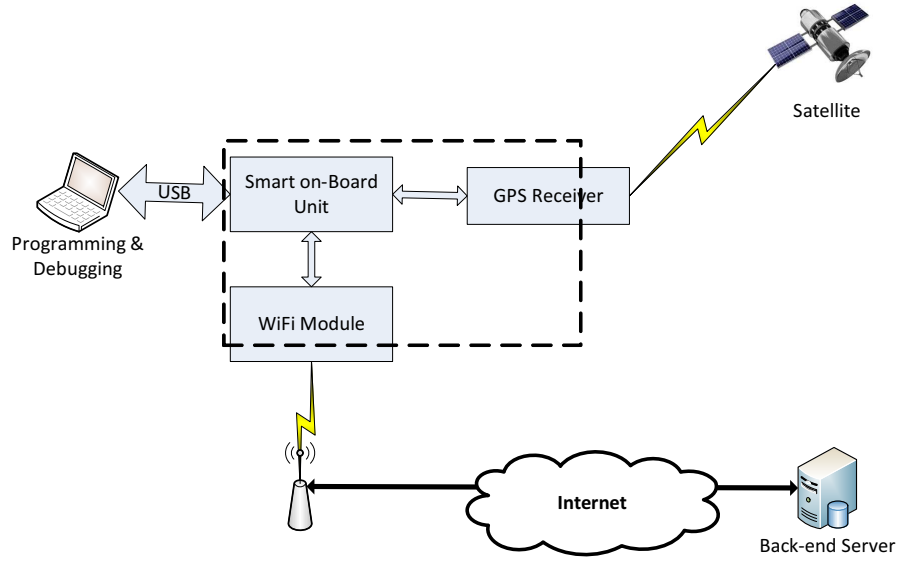

Fig. 1: System High Level Architecture.

\section{A. Smart on-Board Unit (SBU) MAIN COMPONENTS}

Here we provide detailed description about the used hardware modules by the $S B U$ :

1) The Arduino Uno (DEV-11021)Figure 2:which is an open hardware source controller, which is recently being used in many applications, due to its high performance, and easy to deal with. the Arduino Microcontroller board is based on the ATmega328, which has 14 digital input/output pins (of which 6 can be used as PWM outputs), 6 analog inputs, a $16 \mathrm{MHz}$ ceramic resonator,32k Flash Memory, a USB connection, a power jack, and a reset button. It contains everything needed to support the microcontroller; simply connect it to a computer with a USB cable or power it with an AC to DC adapter or battery to get started [15],

2) Arduino GPS Shield (GPS-10710) Figure 3:is a high accuracy GPS receiver, which is used in our system due to its great characteristics and features such as this module can be easily integrated to the Arduino board, GPS-10710 is able to give the vehicle location within a few meters, this GPS module also gives accurate time reading which is an important feature to provide a good distributed synchronization mechanism to our system and all the control messages between the GPS receiver and the Arduino microcontroller are performed using the well-known AT commands standard [15],

3) Arduino Wi-Fi Shield (DEV-11287) Figure 4: this 


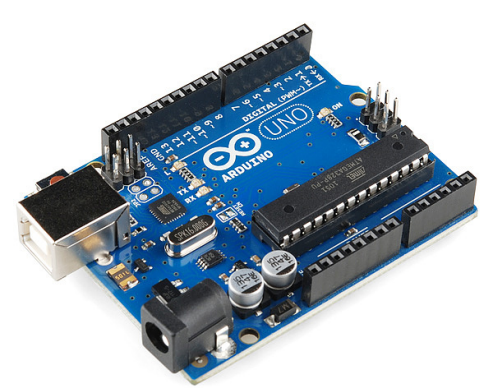

Fig. 2: Arduino Uno Microcontroller [15].

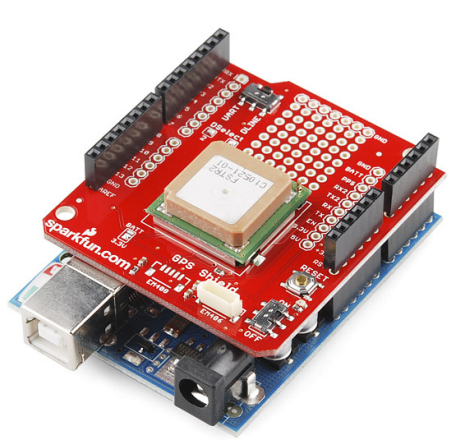

Fig. 3: GPS-10710 Shield Module [15].

Shield allows an Arduino board to connect to the internet using the $802.11 \mathrm{~b} / \mathrm{g}$ wireless specification (WiFi).the shield has an Atmega 32UC3 which provides a network (IP) stack capable of both TCP and UDP. In addition, the shield has an onboard microSD card slot, which is mainly used to locally store the user data on SBU along the trip, before being transferred to the central server via WiFi link. [15].

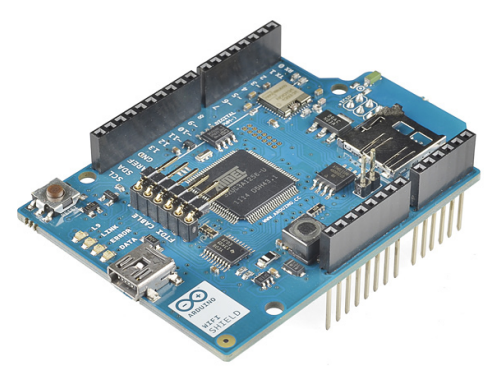

Fig. 4: Wi-Fi Shield Module [15].

\section{B. Web Based Application}

In order to make our Web Application flexible and extendable, we have adapted the REST (RESTful) architecture. And our implemented system has used the three-tier architecture [28]:

1) A front-end which relate to the client side. The user interface is based on a web-browsers application. It contains a responsive web page developed using Hypertext Transfer Markup Language v.5 HTML5, Javascript , JQuery library and Cascading Style Sheet (CSS) whose application is tested on both desktop and smartphone web browsers. This web page uses Asynchronous JavaScript AJAX in order to build bidirectional data flow with middle layer.

2) A middle layer which includes a dynamic PHP program running on top of Apache web server. This program exposes its internal functionality through a RESTful interface towards the front-end and it uses the MySQL native driver for PHP for storing and retrieving data.

3) A back-end containing MySQL database server used to store all known roads in the region, system users, users profiles and user alerts. This component is a relational database that is used to store and retrieve the data. Note that the positioning and speed data are time-stamped according to the UTC time reference.

In RESTful vocabulary things are resources. Each resource is a uniquely addressable entity by a Universal Unique Identifier (URI) attached to it. Moreover, each resource has a representation which can be transferred and manipulated by means of four verbs. These verbs are create, read, update and delete (CRUD).

\section{Functional Testing AND USER ExPERIEnCE}

In order to test the system prototype, we attached our system box to a public transportation vehicle (mini-bus) traveling on the same route/path between two cities; namely, from Tulkarem to Nablus.

we have collocated and locally recorded the trips information (i.e. long., lat., Speed, and timestamp),based on the system model, these information will be transferred via WiFi link to the gateway allocated in the final bus station, by turn, such data will be inserted in the corresponding DataBase. in order to analyze drivers behavior, we are interested in two kind of plots, first plot Figure 5 depicts for each trip, the vehicle speed along the route, while in the second plot as in Figure 6 we show the traveled path along the trip, notice that, the corresponding plot was generated using Open Street Maps (OSM) API [13]. we have considered for analysis Five different trips of the same driver with the same vehicle, it is worth to mention that, these data are collected almost in the same time during a normal working day with almost same weather conditions.

in order to analyze the speed violation, we know that the maximum allowed speed limit on the outside city roads in Palestine is configured to be $90 \mathrm{Km} / \mathrm{h}$, while it is 60 $\mathrm{Km} / \mathrm{h}$ for inside city road segments. by looking to Figure 5 , we notice that, the driver in first trip does not exceed the maximum allowed speed limit; the dotted horizontal line, 


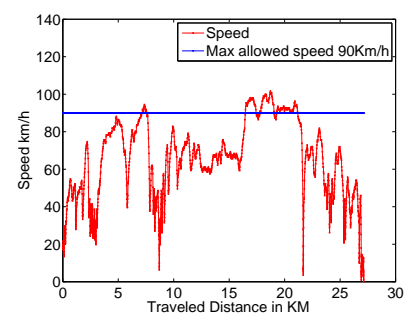

(a)Trip 101

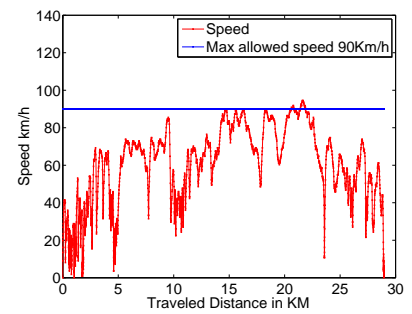

(c)Trip 103

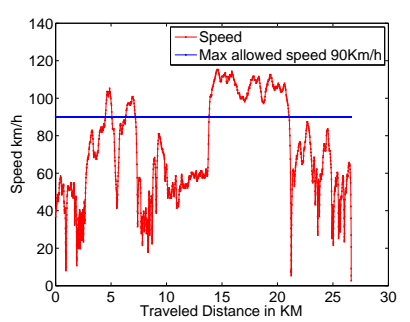

(b)Trip 102

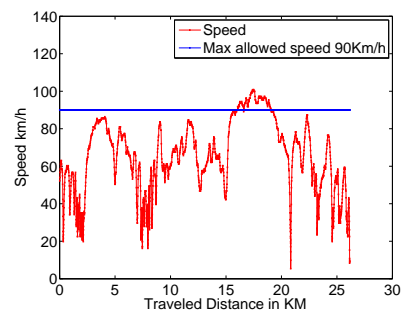

(d)Trip 104

Fig. 5: Driving speed along the route for Four different trips: (a) Trip 101; (b) Trip 102; (c) Trip 103; and, (d) Trip 104.

indicates the $90 \mathrm{Km} / \mathrm{h}$ speed limit, while the connected dotted line is related to $60 \mathrm{Km} / \mathrm{h}$ speed limit. moreover, we could notice that the driver in the second trip 5(b) violates the speed limit frequently in comparison to other trips.on the contrary, the driver in the third trip 5(c) almost did not violates the maximum allowed speed. for more analysis. some related statistics are shown in the table III. it shows for each of the four trips the corresponding, ID, starting date and time, traveled distance in Kilometers unit, traveling trip duration in minutes, average vehicle speed along the whole trip, vehicles maximum reached traveling speed, the maximum legal allowed speed on the outside city road segments, the exceeding max speed duration in Minutes, this metric accumulates the time duration when driver exceeds the speed limit, while the last metric, indicates the percentage of violation duration to the total trip duration. from the statistics in the earlier table, it is easy to notice that the driver violates the speed during the second trip, where the violation percentage is almost $20 \%$ of the time.

Furthermore, Figure 6 shows the followed path for the first four trips, we found that all trips follow the same path along the route, in addition, we indicates on the track (red points) the segments where the driver violates the maximum speed limit, in fact, by referring to the authorized national transportation system, we found that recently, many traffic accidents happened on that segment.

\section{CONCLUSiOnS}

Recently, the demand for developing public transportation management systems(PTMS) using GPS technologies have sharply increased due to the fact that, a well designed PTMS will save human life by monitoring the driver behaviour which in turn will reduce number of times when the driver violate traffic regulations. This paper introduces system composition structure and explains the system software and hardware design. Experiments show that our system is practicable and reliable of data transmissions using WiFi links, with compar-

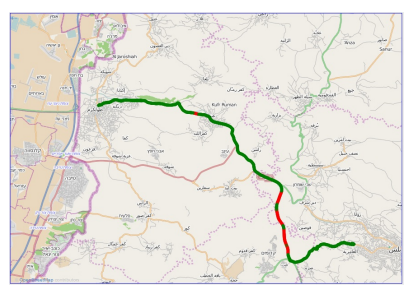

(a)Trip 101

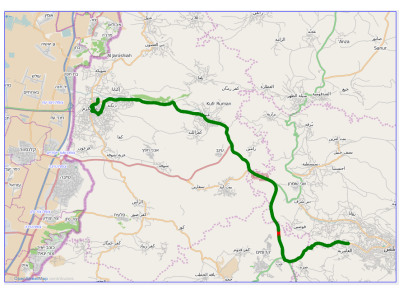

(c)Trip 103

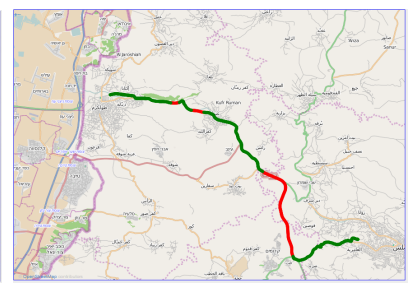

(b)Trip 102

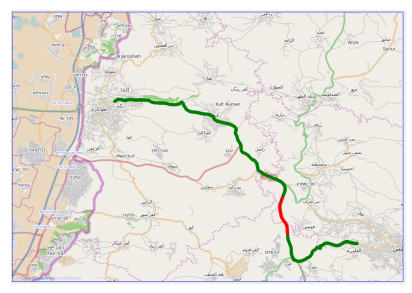

(d)Trip 104
Fig. 6: Traveled Path along the route for Four different trips: (a) Trip 101; (b) Trip 102; (c) Trip 103; and, (d) Trip 104.

ison with the others management systems based on GPRS and GPS technology, it is greatly reduced the operating price. In our system, the Open Street Maps plays a major rule during monitoring, visualization and identifying the maximum allowed speed for each road segment along with the traveled route. Ongoing work is to enable the system for supporting online tracking and monitoring services, by using mobile data networks.

\section{REFERENCES}

[1] S. Tarapiah, S. Atalla, N. Muala, and S. Tarabeh, "Offline public transportation management system based on GPS/WiFi and open street maps," in CICSyN2014, 6th Int Conference on Computational Intelligence, Communication Systems and Networks (CICSyN2014), Tetovo, Macedonia, the former Yugoslav Republic of, May 2014.

[2] S. Tarapiah, R. AbuHania, and D. J. Islam Hindi, "Applying web based gps/gprs ticketing and tracking mechanism to reduce traffic violation in developing countries," in The International Conference on Digital Information Processing, E-Business and Cloud Computing (DIPECC2013). The Society of Digital Information and Wireless Communication, 2013, pp. 102-106.

[3] S. Tarapiah, S. Atalla, and R. AbuHania, "Smart on-board transportation management system using gps/gsm/gprs technologies to reduce traffic violation in developing countries," International Journal of Digital Information and Wireless Communications (IJDIWC), vol. 3, no. 4, pp. 96-105, 2013.

[4] S. Tarapiah, S. Atalla, and B. Alsayid, "Smart On-Board transportation management system Geo-Casting featured," in International Conference on Computer Information Systems 2014 (ICCIS-2014), Hammamet, Tunisia, Jan. 2014.

[5] G. A. Giannopoulos, "The application of information and communication technologies in transport," European Journal of Operational Research, vol. 152, no. 2, pp. 302-320, 2004.

[6] M. Al-Rousan, A. Al-Ali, and K. Darwish, "Gsm-based mobile telemonitoring and management system for inter-cities public transportations," in Industrial Technology, 2004. IEEE ICIT'04. 2004 IEEE International Conference on, vol. 2. IEEE, 2004, pp. 859-862.

[7] M. Popa and B. Suta, "A solution for tracking a fleet of vehicles," in Telecommunications Forum (TELFOR), 2011 19th. IEEE, 2011, pp. $1558-1561$.

[8] C. Koukourlis, S. Spyridakis, and N. Kokkalis, "On the design of a fleet monitoring system with reduced power consumption," Electrical 


\begin{tabular}{|c|c|c|c|c|c|}
\hline Trip ID & 1 & 2 & 3 & 4 & 5 \\
\hline Starting Time & 1_19_20149_22am & 1_20_2014 8_16am & 1_21_20149_37am & 1_22_2014 8_28am & 1_23_2014 9_34am \\
\hline Distance $(\mathrm{Km})$ & 27.122 & 26.65 & 28.95 & 26.15 & 25.03 \\
\hline Duration (minutes) & 28.62 & 25.4 & 34.48 & 26.36 & 26.8 \\
\hline Average Speed $(\mathrm{Km} / \mathrm{H})$ & 56.86 & 62.96 & 50.37 & 59.5 & 56.03 \\
\hline Maximum Speed & 101.7 & 115.2 & 94.5 & 100.8 & 109.8 \\
\hline Maximum Allowed Speed $(\mathrm{km} / \mathrm{h})$ & 90 & 90 & 90 & 90 & 90 \\
\hline Exceeding Max allowed Speed Duration & 2.85 & 4.95 & 0.37 & 1.9 & 3.43 \\
\hline Violating Speed (\%) & 09.69 & 19.49 & 01.06 & 07.20 & 12.81 \\
\hline
\end{tabular}

TABLE III: Trips Related Statistics.

Engineering, vol. 84, no. 4, pp. 203-210, 2002. [Online]. Available: http://dx.doi.org/10.1007/s00202-002-0120-z

[9] I. M. Almomani, N. Y. Alkhalil, E. M. Ahmad, and R. M. Jodeh, "Ubiquitous gps vehicle tracking and management system," in Applied Electrical Engineering and Computing Technologies (AEECT), 2011 IEEE Jordan Conference on. IEEE, 2011, pp. 1-6.

[10] I. A. H. Eltoum and M. Bouhorma, "Velocity based tracking and localization system using smartphones with gps and gprs/3g." International Journal of Computer Applications, vol. 76, 2013.

[11] J. Biagioni, T. Gerlich, T. Merrifield, and J. Eriksson, "Easytracker: automatic transit tracking, mapping, and arrival time prediction using smartphones," in Proceedings of the 9th ACM Conference on Embedded Networked Sensor Systems. ACM, 2011, pp. 68-81.

[12] Y. Lou, C. Zhang, Y. Zheng, X. Xie, W. Wang, and Y. Huang, "Mapmatching for low-sampling-rate gps trajectories," in Proceedings of the 17th ACM SIGSPATIAL International Conference on Advances in Geographic Information Systems. ACM, 2009, pp. 352-361.

[13] "Open Street Map," 2014. [Online]. Available: http://www.openstreetmap.org/

[14] "Javascript Library Open Layer," 2014. [Online]. Available: http://openlayers.org/

[15] "SparkFun Electronics," 2013. [Online]. Available: www.sparkfun.com 